\title{
Pacific
}

Journal of

Mathematics

THE NUMBER OF LATTICE POINTS WITHIN A CONTOUR AND VISIBLE FROM THE ORIGIN

Douglas Austin Hensley

Volume 166 No. 2

December 1994 


\section{THE NUMBER OF LATTICE POINTS WITHIN A CONTOUR AND VISIBLE FROM THE ORIGIN}

\section{Doug Hensley}

The main result is an estimate for the number $P(r)$ of relatively prime pairs $(a, b)$ of integers within a contour. When specialized to the contour $x^{2}+y^{2}=r$ this estimate gives

$$
\begin{aligned}
& P(r)=(6 / \pi) r \\
& +\left(\text { without the RH, } O_{\epsilon}\left(r^{1 / 2} \exp \left(-(\log r)^{(3 / 5)+\epsilon}\right)\right)\right. \\
& \text { or with the RH } \left.O_{\epsilon} r^{(51+\epsilon) / 100}\right) \text {. }
\end{aligned}
$$

A similar estimate, with the same sort of error, is obtained for the number of relatively prime pairs $(a, b)$ of positive integers so that $a b \leq r$. The error term for a general contour depends on the maximal value of the radius of curvature of the bounding contour.

1. Introduction. The number $R(r)$ of integer pairs $(a, b)$ for which $a^{2}+b^{2} \leq r$ is known to satisfy

$$
R(r)=\pi r+O_{\epsilon}\left(r^{\theta+\epsilon}\right)
$$

where $1 / 4 \leq \theta \leq 7 / 22$. The main result here is an estimate of the number $P(r)$ of relatively prime integer pairs $(a, b)$ within a contour. When specialized to the contour $x_{1}^{2}+x_{2}^{2}=r$, it gives

$$
P(r)=(6 / \pi) r+O_{\epsilon}\left(r^{1 / 2} \exp \left(-(\log r)^{(3 / 5)+\epsilon}\right)\right) .
$$

If the Riemann Hypothesis ( $\mathrm{RH}$ ) holds, this improves to

$$
P(r)=(6 / \pi) r+O_{\epsilon}\left(r^{(51 / 110)+\epsilon}\right) .
$$

If we assume further that the correct value of $\theta$ in the circle problem is $1 / 4$, then the exponent $51 / 110$ becomes $9 / 10$. We give a 
comparable estimate for the divisor problem (lattice points under a hyperbola) in section 3 .

The first estimate, which does not depend on any unproven hypotheses, holds uniformly over a wide range of convex contours enclosing the origin. The key parameter is the maximum radius of curvature $\rho$. The number of lattice points inside a square with sides of length $r$ parallel to the coordinate axes is $r^{2}+O(r)$. The error term cannot be improved because a whole rank of lattice points is lined up along the boundary of the square, and the slightest change in $r$ can move the boundary line to the other side of this rank of lattice points. The same phenomenon obstructs the estimation of lattice point count inside arbitrary regions whenever the region contains a particularly straight segment at any slope. The basic theorem on lattice points in $\Re^{2}$ within a region $A$ is due to van der Corput, and with explicit constants and an elementary proof, to Chaix [3]. It takes this difficulty into account by framing the estimate in terms of the maximum straightness of the bounding curve, that is, in terms of the maximal radius of curvature $\rho=\rho(A)$ :

Theorem (VAN DER CoRPut, ChAIX). There exists an explicitly computable constant $C>0$ such that if $A$ is a convex region in $\Re^{2}$ with maximal radius of curvature $\rho(A)>0$, then

$$
\mid \text { Lattice Count }(A)-\text { Area }(A) \mid \leq C(1+\rho(A))^{2 / 3} \text {. }
$$

The exponent is best possible, although in special cases such as that of a circle with center at a lattice point the exponent can be improved some. For the circle, the best known constant, due to Iwaniec and Mozzochi [2], is $7 / 11+\epsilon$. It is known that this exponent cannot be less than $1 / 2$. There is no real consensus, nor is there strong numerical evidence, on whether or not the true value is $1 / 2+$ $\epsilon$.

The results given here for the number of visible lattice points (points with relatively prime integer coordinates) improve on an unpublished result of Biagiolli, in which one had $\left(6 / \pi^{2}\right)$ Area $(A)+$ $O(\rho)$ for reasonable contours. The general idea is, in both cases, inclusion and exclusion using the Möbius function $\mu(d)$, but this time with a trick to take advantage of the smooth contour. While it is not standard notation, we shall for technical convenience exclude 
0 from all lattice point counts from now on. This will not affect the conclusions. (The trouble with counting the origin is that inclusion and exclusion fails. A domain far from the origin poses a related problem, which applies to all the lattice points in the domain. Large divisors can sift the set of visible points down to an empty remnant.)

Let $A$ be a domain in $\Re^{2}$, including 0 , with area $|A|$ and maximal radius of curvature $\rho=\rho(A)$ on its (smooth) boundary. More precisely, the boundary of $A$ should at all points have a unique unit tangent vector when traced counterclockwise, and for any arc of length $s$ along this boundary, the cumulative change in direction for this vector should be at least $s / \rho(A)$.

Let $N(A)=\sup \left\{\left|\left(x_{1}, x_{2}\right)\right|:\left(x_{1}, x_{2}\right) \in A\right\}$ be the maximum distance from the origin to the boundary of $A$. Clearly $A$ lies within any circle of radius $\rho(A)$ tangent to $A$ and convex in the same direction, so that $N(A) \leq 2 \rho(A)$. We may as well assume $\rho$ large (details later), and we do. Let $R(A)$ denote the number of lattice points exclusive of the origin, or integer pairs $(a, b) \neq 0$ in $A$, and let $P(A)$ denote the number of relatively prime pairs among these. Then with $t A:=\left\{t\left(x_{1}, x_{2}\right):\left(x_{1}, x_{2}\right) \in A\right\}$, we have by inclusion and exclusion

$$
P(A)=\sum_{d=1}^{N(A)} \mu(d) R\left(d^{-1} A\right)
$$

For an arbitrary domain $B$, let $E(B):=R(B)-|B|$. (Later, we will have occasion to use a different definition of $E$ in connection with the divisor problem. The idea is that $E$ denotes the difference between the actual number of nonzero lattice points enclosed and the expected number, which is in most cases the area.)

Let $\left(t_{1}, t_{2}, \ldots t_{k}\right)=\left(t_{1}(A), t_{2}(A), \ldots t_{k(A)}(A)\right)$ be the increasing sequence of real numbers $t \in(0,1]$ for which the boundary of $t A$ contains a lattice point. Let $\tau_{j}=1 / t_{j}$. Let $I_{j}(A)$ denote the interval $\left(\tau_{j+1} . . \tau_{j}\right]$. Then for any $y, 1<y<N(A)$, and with the convention that if $\tau_{j+1}<y<\tau_{j}$ then $I_{j}$ is truncated at the lower end by $y$, we 
have

$$
\text { (2) } \begin{aligned}
P(A)=|A| \sum_{d \leq N(A)} d^{-2} \mu(d) & +\sum_{d \leq y} \mu(d) E\left(d^{-1} A\right)+ \\
+ & \sum_{\tau_{j}>y} E\left(\tau_{j}^{-1} A\right) \sum_{d \in I_{j}(A)} \mu(d)
\end{aligned}
$$

This is the trick. Different methods suit different terms.

The first term here is

$$
\text { Main }+E_{0}=\frac{6}{\pi^{2}}|A|+O_{\epsilon}\left(\frac{|A|}{\rho(A)} \exp \left(-(\log \rho(A))^{(3 / 5)+\epsilon}\right)\right)
$$

from the well known estimate [5] for the Möbius sum

$$
M(x):=\sum_{d \leq x} \mu(d)=O_{\epsilon}\left(x \exp \left(-(\log x)^{(3 / 5)-\epsilon}\right)\right) .
$$

To estimate the second term one simply uses the van der CorputChaix [3] estimate

$$
E(A)=O\left(1+\rho(A)^{2 / 3}\right)
$$

which holds uniformly over all domains $A$. In this second term, $d \leq N(A) \ll \rho(A)$, the radius of curvature of $d^{-1} A$ will be at least comparable to 1 , and the " 1 " can be omitted in the estimate above. For the last two terms we can take advantage of the hard-core number theoretic bounds on $M(x): M(x) \ll x \exp \left(-\log ^{3 / 5-\epsilon} x\right)$, or on the Riemann Hypothesis, $M(x) \ll x^{\frac{1}{2}+\epsilon}$. This latter estimate, if true, is roughly best possible. Odlyzko and teRiele [6] have even shown that Merten's conjecture, to the effect that $\left|\sum_{d \leq x} \mu(d)\right| \leq$ $x^{1 / 2}$ is false.

In the next section we discuss the various error terms and prove a theorem for general, reasonably rounded, convex contours enclosing the origin. In section three, as mentioned previously, we count relatively prime pairs for which the product is bounded by $r^{2}$.

2. The general case. For the routine text of this section, we abbreviate $\rho(A)$ to $\rho$. There are several error terms to control. We 
have an estimate for $E_{0}$ from section 1 . For $E_{1}$ we have the simple estimate

$$
E_{1}=O\left(\sum_{d \leq y} d^{-2 / 3} \rho^{2 / 3}\right)=O\left(y^{1 / 3} \rho^{2 / 3}\right)
$$

from (3). Now again with the convention that replaces $\tau_{j+1}$ with $y$ if $\tau_{j+1}<y<\tau_{j}$,

$$
\begin{aligned}
& E_{2}=\sum_{\tau_{j}>y} E\left(\tau_{j}^{-1} A\right)\left(M\left(\tau_{j}\right)-M\left(\tau_{j+1}\right)\right) \\
& =O_{\epsilon}\left(\rho^{2 / 3} \sum_{\tau_{j}>y} \tau_{j}^{1 / 3} \exp \left(-\log ^{(3 / 5)-\epsilon} \tau_{j}\right)\right)
\end{aligned}
$$

or on the RH,

$$
E_{2}=O_{\epsilon}\left(\rho^{2 / 3} \sum_{\tau_{j}>y} \tau_{j}^{\epsilon-(1 / 6)}\right)
$$

Finally, integrating by parts gives

$$
\begin{aligned}
E_{3} & =2|A| \sum_{\tau_{j}>y} \int_{\tau_{j+1}}^{\tau_{j}} s^{-3}\left(M(s)-M\left(\tau_{j+1}\right)\right) d s \\
& =O_{\epsilon}\left(|A| y^{-1} \exp \left(-\log { }^{(3 / 5)-\epsilon}(y)\right)\right) .
\end{aligned}
$$

On the $\mathrm{RH}$, the same approach gives

$$
E_{3}=O_{\epsilon}\left(|A| y^{-(3 / 2)+\epsilon}\right) \text {. }
$$

Now fix $\epsilon>0$, put aside the $\mathrm{RH}$, and take

$$
y=\rho \exp \left(-(1 / 2) \log ^{(3 / 5)-\epsilon} \rho\right) .
$$

Then for $\rho$ sufficiently large,

$$
\log y \geq(11 / 12) \log ^{(3 / 5)-\epsilon} \rho .
$$

This condition specifies "sufficiently large" $\rho$ from the introduction. Now it will be convenient to set $\delta:=\frac{3}{5}-\epsilon$.

Continuing with the analysis of $E_{2}$, from (4) and (6) we have

$$
E_{2}=O_{\epsilon}\left(\rho^{2 / 3} \exp \left(-(11 / 12) \log ^{\delta} \rho\right) \sum_{\tau_{j}>y} \tau_{j}^{1 / 3}\right)
$$


If, in this last sum, we count the terms by multiplicity according to the number of lattice points on the boundary of $t_{j} A$, this can only serve to increase the sum. With this convention, though, we have the sum in (7) is

$$
\sum_{\left\{j: t_{j}<\rho^{-1} \exp \left(\frac{1}{2} \log ^{\delta} \rho\right)\right\}} t_{j}^{-1 / 3} \ll \sum_{\lambda \in Z^{2} \cap \rho^{-1}} \sum_{\exp \left(\frac{1}{2} \log ^{\delta} \rho\right) A}|\lambda|^{-1 / 3} \rho^{1 / 3}
$$

and on replacing the scaled copy of $A$ with a disk $D$ about the origin of radius $4 \exp \left(\frac{1}{2} \log ^{\delta} \rho\right)$, this sum increases to $\ll \rho^{1 / 3} \sum_{\lambda \in D}|\lambda|^{-1 / 3} \ll$ $\rho^{1 / 3} \exp \left(\frac{5}{6} \log ^{\delta} \rho\right)$ and it follows immediately that

$$
E_{2}=O_{\epsilon}\left(\rho \exp \left(-\frac{1}{12} \log ^{\delta} \rho\right)\right) .
$$

With our choice of $y$, the bound (5) on $E_{3}$ reduces to

$$
E_{3}=O_{\epsilon}\left(\exp \left(-\frac{5}{12} \log ^{\delta} \rho\right) \rho^{-1}|A|\right)=O_{\epsilon}\left(\rho \exp \left(-\frac{5}{12} \log ^{\delta} \rho\right)\right)
$$

which is less than the tolerance allowed for in other error term estimates. With this same choice of $y, E_{1}=O_{\epsilon}\left(\rho \exp \left(-(1 / 6) \log ^{\delta} \rho\right)\right)$. In view of the stronger bound on $E_{0}$, this establishes

THEOREM 1. There exists a constant $C>0$ such that for all fixed $\epsilon>0$, uniformly over $A$ containing the origin and for which $\rho(A) \geq C$,

$$
P(A)=\frac{6}{\pi^{2}}|A|+O_{\epsilon}\left(\rho(A) \exp \left(-\log ^{(3 / 5)-\epsilon}(A)\right)\right) .
$$

Assuming the Riemann Hypothesis gave us

$$
E_{0}=O_{\epsilon}\left(\rho^{\epsilon-3 / 2}|A|\right), E_{1}=O\left(y^{1 / 3} \rho^{2 / 3}\right)
$$

$$
E_{3}=O_{\epsilon}\left(|A| y^{\epsilon-3 / 2}\right), \quad \text { and } \quad E_{2}=O_{\epsilon}\left(\rho^{2 / 3} \sum_{\tau_{j}>y} \tau_{j}^{\epsilon-(1 / 6)}\right) .
$$

Proceeding as before with $E_{2}$ we get, this time on taking $y=\rho^{4 / 5}$, that 
$\left(9^{\prime}\right)$

$$
E_{2}=O_{\epsilon}\left(\rho^{2 / 3} \sum_{|\lambda| \ll \rho^{1 / 5}}(\rho /|\lambda|)^{\epsilon-1 / 6}\right)=O_{\epsilon}\left(\rho^{(14 / 15)+\epsilon}\right) .
$$

This now gives

THEOREM 2. If the Riemann hypothesis is correct, then with the same hypotheses and uniformity as in theorem 1,

$$
P(A)=\frac{6}{\pi^{2}}|A|+O_{\epsilon}\left(\rho(A)^{(14 / 15)+\epsilon}\right) .
$$

REMARK. Any upper bound $\Theta<1$ for the real part of a zero of $\zeta(s)$ leads to a similar bound on the error in $P(A)$ with an exponent less than one on $\rho(A)$. Stronger bounds for $E(A)$ for particular contours likewise yield improved estimates, but only in conjunction with the Riemann hypothesis.

3. The circle and divisor problems. Although the region under a hyperbola does not fit the premises of theorems 1 and 2, a similar result holds. We give details only for the case of the divisor problem, but the same Riemann-hypothesis-conditional estimate for the number of points in a circle about the origin of radius $\rho$ holds with virtually the same proof. We begin with the recent sharp estimate [2] for the number of lattice points in the region $A_{\rho}:=\left\{\left(x_{1}, x_{2}\right): \frac{1}{2} \leq x_{1}, x_{2}, x_{1} x_{2} \leq \rho^{2}\right\}$. They give (with $C=2 \gamma-1)$

$$
R\left(A_{\rho}\right)=2 \rho^{2} \log \left(\rho^{2}\right)+C \rho^{2}+O_{\epsilon}\left(\rho^{(7+\epsilon) / 11}\right) .
$$

Though this estimate does not have the exact form of "pointcount= $=$ area + small error", the ideas of section 2 go th rough. The analogue to (1) is

$$
\begin{aligned}
P(\rho) & =\sum_{d \leq \rho} \mu(d) R\left(d^{-1} \rho\right) \\
& =\sum_{1 \leq d \leq \rho} \mu(d)\left(4(\rho / d)^{2} \log (\rho / d)+C \rho^{2} / d^{2}+E(\rho / d)\right) \\
& =\sum_{d \leq \rho} \mu(d)(F(\rho / d)+E(\rho / d)) \quad \text { say }
\end{aligned}
$$


where now $R(z):=R\left(\left\{\left(x_{1}, x_{2}\right): 1 / 2 \leq x_{1}, x_{2}, x_{1} x_{2} \leq z^{2}\right\}\right)$ and $E(z):=R(z)-4 z^{2} \log z-C z^{2}$. By[2], $E(z)=O_{\epsilon}\left(z^{(7+\epsilon) / 11}\right)$. As in (2), with $P(\rho):=P\left(A_{\rho}\right)$ etc., this gives

$$
\begin{aligned}
P(\rho) & =\sum_{d \leq \rho} \mu(d)(F(\rho / d)+E(\rho / d)) \\
& =\sum_{d \geq 1} \mu(d) F(\rho / d)-\sum_{d>\rho} \mu(d) F(\rho / d)+\sum_{d \leq \rho} \mu(d) E(\rho / d) .
\end{aligned}
$$

Now put $E_{0}=-\sum_{d>\rho} \mu(d) F(\rho / d)$. Put $\bar{E}=\sum_{d \leq \rho} \mu(d) E(\rho / d)$. Then for arbitrary $y, 1<y<\rho$ we have

$$
\bar{E}=\sum_{d \leq y} \mu(d) E(\rho / d)+\sum_{y<d \leq \rho} \mu(d) E(\rho / d) .
$$

Let $\left(t_{1}, t_{2}, \ldots t_{k} \leq 1\right)$ be the successive values at which $R\left(t_{j} \rho\right)$ increases. Let $\tau_{j}=1 / t_{j}$. This time, $t_{j} \rho$ runs through the integers from 1 to $\left[\rho^{2}\right]$, so that $t_{j}=j^{1 / 2} \rho^{-1}$ and $\tau_{j}=\rho j^{-1 / 2}$. Put $I_{j}(\rho):=$ $\left(\tau_{j+1}, \tau_{j}\right]$, except that in the least interval which meets $(y, \infty)$ we replace $\tau_{j+1}$ with $y$. As before we now discuss separately the cases in which $\tau_{j} \leq y,>y$. For the case $\tau_{j}>y$ we take note of the fact that $R(\rho / d)=R\left(\rho / \tau_{j}\right)$ whenever $\tau_{j+1}<d \leq \tau_{j}$. As in (2), we break down $\bar{E}$ further with

$$
\begin{aligned}
\bar{E}= & \sum_{d \leq y} \mu(d) E(\rho / d)+\sum_{\tau_{j}>y} \sum_{d \in I_{j}} \mu(d) E\left(\rho / \tau_{j}\right) \\
& +\sum_{\tau_{j}>y} \sum_{d \in I_{j}} \mu(d)\left(F\left(\rho / \tau_{j}\right)-F(\rho / d)\right)
\end{aligned}
$$

so that $P(\rho)=\sum_{d \geq 1} \mu(d) F(\rho / d)+E_{0}+E_{1}+E_{2}+E_{3}$ say. Now

$$
\begin{aligned}
& \text { (16) } E_{0}=-\sum_{d>\rho} \mu(d)\left(4 \frac{\rho^{2}}{d^{2}} \log \left(\frac{\rho}{d}\right)+C \frac{\rho^{2}}{d^{2}}\right) \\
& \ll \int_{\rho}^{\infty} \rho^{2}(1+\log s) s^{\epsilon-5 / 2} d s \ll \rho^{\epsilon+1 / 2} .
\end{aligned}
$$

From [2],

$$
E_{1} \ll \sum_{d \leq \rho^{4 / 5}}\left(\frac{\rho}{d}\right)^{\epsilon+7 / 11} \ll \rho^{51 / 55+\epsilon}
$$


Again the estimation of $E_{2}$ is the main issue. We have

$$
E_{2} \ll \sum_{\tau_{j}>y}\left|E\left(\rho / \tau_{j}\right)\right|\left|M\left(\tau_{j+1}\right)-M\left(\tau_{j}\right)\right| .
$$

Taking $y=\rho^{4 / 5}$, this gives

$$
\begin{aligned}
E_{2} & \ll \rho^{1 / 2+\epsilon} \sum_{j<\rho^{2 / 5}} E\left(j^{1 / 2}\right) \rho^{1 / 2+\epsilon} j^{-1 / 4} \\
& \ll \rho^{1 / 2+\epsilon} \sum_{j<\rho^{2 / 5}} j^{7 / 22-1 / 4+\epsilon}<\rho^{51 / 55+2 \epsilon} .
\end{aligned}
$$

Now

$$
\begin{aligned}
E_{3} & =\sum_{\tau_{j}>y} \int_{\tau_{j+1}}^{\tau_{j}}\left(F\left(\rho / \tau_{j}\right)-F(\rho / s)\right) d M(s) \\
& =-\sum_{j<\rho^{2 / 5}} \int_{\tau_{j+1}}^{\tau_{j}} s^{-2} F^{\prime}(\rho / s)\left(M(s)-M\left(\tau_{j}\right)\right) d s \\
& \ll \sum_{j \leq \rho^{2 / 5}} \int_{\tau_{j+1}}^{\tau_{j}} s^{\epsilon-3 / 2} F^{\prime}(\rho / s) d s \\
& \ll \int_{\rho^{2 / 5}}^{\rho} s^{\epsilon-3 / 2}(\rho / s) \log (\rho / s) d s \ll \rho^{2 / 5+\epsilon} .
\end{aligned}
$$

We now have the bounds $E_{0} \ll \rho^{\epsilon+1 / 2}, E_{1} \ll \rho^{51 / 55+\epsilon}, E_{2} \ll \rho^{51 / 55+2 \epsilon}$, and $E_{3} \ll \rho^{2 / 5+\epsilon}$. Thus $E_{j} \ll \rho^{51 / 55+2 \epsilon}$ for all four error terms $E_{j}$, so that (since $\epsilon$ is arbitrary)

$$
P(\rho)=\sum_{d \geq 1} \mu(d) F(\rho / d)+O_{\epsilon}\left(\rho^{\epsilon+51 / 55}\right) .
$$

An elementary calculation now gives

$$
\sum_{d \geq 1} \mu(d) F(\rho / d)=\frac{24}{\pi^{2}} \rho^{2} \log \rho+\frac{6}{\pi^{2}}(2 \gamma-1) \rho^{2}-\frac{144}{\pi^{4}} \zeta^{\prime}(2)
$$

which gives our final result:

THEOREM 3. If the Riemann hypothesis is true, then the number of pairs $P(\rho)$ of relatively prime positive integers with product no larger than $\rho^{2}$ as $\rho \rightarrow \infty$ is given by

$$
P(\rho)=\frac{24}{\pi^{2}} \rho^{2} \log \rho+\frac{6}{\pi^{2}}(2 \gamma-1) \rho^{2}-\frac{144}{\pi^{4}} \zeta^{\prime}(2)+O_{\epsilon}\left(\rho^{\epsilon+51 / 55}\right) .
$$


REMARKS. If we assume further that the correct exponent in the divisor or circle problem is $(1 / 4)+\epsilon$, then $51 / 55$ in theorem 3 can be reduced to $9 / 10$. Even moderately convincing numerical evidence for the true rate of growth in $\max _{\rho \leq x} P\left(A_{\rho}\right)$, say for the circle, is not easily amassed. The best published studies depend on an algorithm that requires $O\left(\rho^{1 / 2}\right)$ evaluations of the integer part of a square root. Vinogradov, and later in a sharper form Chaix [3] gave an elementary proof that $R\left(A_{\rho}\right)=\left|A_{\rho}\right|+O\left(\rho^{1 / 3}\right)$. Their ideas can be adapted to give an algorithm which requires $O\left(\rho^{1 / 3} \log \rho\right)$ evaluations of the integer part of a square root. (The log factor can be eliminated but at the cost of an impractically large implicit constant.) Even so, finding $P\left(A_{\rho}\right)$ requires $O\left(\rho^{1 / 2}\right)$ evaluations of the integer part of a square root. Small scale computation fails to indicate a dramatic difference in the behaviors of the error term for $R$ and $P$.

\section{REFERENCES}

[1] A. Biagiolli, private communication.

[2] H. Iwaniec and C.J. Mozzochi, On the divisor and circle problem, (to appear) (39 pp).

[3] H. Chaix, Démonstration élémentaire d'un théorème de Van der Corput, C.R. Acad. Sc. Paris, 275 (1972) Series A, 883-885.

[4] W. Fraser and C. C. Gotlieb, A calculation of the number of lattice points in the circle and sphere, Math. Comp., 16 (1962), 282-290.

[5] A. Ivić, The Theory of the Riemann Zeta Function with Applications, Wiley \& Sons, New York 1985.

[6] A. Odlyzko and H. te Riele, Disproof of the Mertens conjecture, J. Reine u. Angew. Math., 357 (1985), 138-160.

Received September 28, 1992, revised December 21, 1993, and accepted for publication October 17, 1994.

Texas A \& M UNIVERsity

College Station, TX 77843-3368 



\section{PACIFIC JOURNAL OF MATHEMATICS}

Volume $166 \quad$ No. $2 \quad$ December 1994

Geometric aspects of Bäcklund transformations of Weingarten

submanifolds

STEVEN BUYSKE

Multipliers between invariant subspaces of the backward shift

225

ROBERT BRUCE CROFOOT

The Cauchy integral, analytic capacity and subsets of quasicircles

XIANG FANG

The number of lattice points within a contour and visible from the origin 295

Douglas Austin Hensley

On flatness of the Coxeter graph $E_{8}$

305

MASAKI IZUMI

Immersions up to joint-bordism

GUI SONG LI

Generalization of the Hilbert metric to the space of positive definite matrices

CARlangelo Liverani and Maciej WojtKowski

Periodicity, genera and Alexander polynomials of knots

SWATEE NAIK

On divisors of sums of integers. $\mathrm{V}$

ANDRÁS SÁRKÖZY and CAMERON LEIGH STEWART

Approximately inner automorphisms on inclusions of type $\mathrm{III}_{\lambda}$-factors

CARL WINSLøW

Correction to: "A convexity theorem for semisimple symmetric spaces"

KARL-HERMANN NEEB

Correction to: "Periodic points on nilmanifolds and solvmanifolds"

EDWARD KEPPELMANN

Correction to: "Partially measurable sets in measure spaces" 